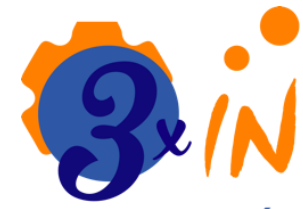

INVESTIGACIÓN e INNOVACIÓN en INGENERÍAS ISSN2344-8652

Open Access

Recibido:

5 abril de 2018

Aceptado:

11 julio de 2018

Publicado:

11 diciembre de 2018

Correspondencia:

cdiaz@tecnologicocomfenalco.edu.c

DOI:

https://doi.org/10.17081/invinno.7.1 .3129

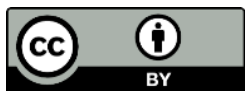

(c) Copyright: Investigación e Innovación en Ingenierías

\section{Carácterización de la especie Chrysobalanus Icaco como alternativa de reforestación para mitigar procesos de erosión costera. Estudio de caso sostenibilidad ambiental de la especie en el departamento de Bolívar-Colombia}

\section{Characterization of the Chrysobalanus Icaco species as a reforesta- tion alternative to mitigate coastal erosion processes. Case study environmental sustainability of the species in the department of Bolívar-Colombia}

\author{
Claudia Díaz Mendoza (iD, Ledis Paola Ayola Mendoza iD, Yesid Alberto Morelo \\ González (iD, Iván Díaz Gómez iD, Yunelis Burgos \\ Fundación Universitaria Tecnológico Comfenalco Cartagena, Colombia
}

Resumen

Objetivo: Estudiar la especie Chrysobalanus Icaco como alternativa de reforestación para manejo de erosión, teniendo en cuenta el potencial de la especie para la conservación y protección de suelos. Metodología: La metodología que manejó el estudio contempló métodos y técnicas inscritas en investigaciones de campo, descriptiva y correlacional. Resultados y conclusiones: El análisis indicó que la especie puede ser utilizada en el departamento para disminuir procesos erosivos. Sin embargo, las condiciones ambientales deben adecuarse con fertilizantes ricos en oligoelementos para la reforestación con Icaco con fines de aprovechamiento agroindustrial, manejo de erosión y conservación de la especie.

Palabras claves: Problemas ambientales, micronutrientes, macronutrientes, método de restauración, conservación, condiciones climatológicas, fertilizantes, servicios ambientales, proceso de germinación, dunas Costeras.

\section{Abstract}

Objective: To study the species Chrysobalanus Icaco as an alternative of reforestation for erosion management, taking into account the potential of the species for the conservation and protection of soils. Methodology: The methodology that handled the study contemplated methods and techniques registered in field research, descriptive and correlational. Results and conclusions: The analysis indicated that the species can be used in the department to reduce erosion processes. However, environmental conditions must be adapted with fertilizers rich in trace elements for reforestation with Icaco for the purpose of agro-industrial use, erosion management and conservation of the species.

Keywords: Environmental problems, micro Nutrients, macro Nutrients, restoration method, conservation, weather condition, fertilizers, environmental services, germination process, coastal dunes.

Como citar (IEEE): C. Diaz - Mendoza, L. Ayola - Mendoza, Y. Morelo - Gonzalez, I. Díaz - Gómez, Y- Burgos - Alarcón, y J. Bethsaid Pedroza - Rojas, "Carácterización de la especie Chrysobalanus Icaco como alternativa de reforestación para mitigar procesos de erosión costera. Estudio de caso sostenibilidad ambiental de la especie en el departamento de Bolívar-Colombia", Investigación e Innovación en Ingenierías, vol. 7, nº. 1, 2019. DOI: https://doi.org/10.17081/invinno.7.1.3129 
Carácterización de la especie Chrysobalanus Icaco como alternativa de reforestación para mitigar procesos de erosión costera. Estudio de caso sostenibilidad ambiental de la especie en el departamento de Bolívar-Colombia

\section{Introducción}

La erosión es un proceso físico que consiste en el desprendimiento y arrastre de materiales del suelo, debido a factores naturales o antrópicos que afectan el sistema funcional del suelo [1]. Existen varios tipos de erosión, y la erosión costera es uno de ellos. En Cartagena de Indias representa uno de los principales problemas ambientales; pues, la mayor parte de la zona costera del departamento de Bolívar le corresponde a este distrito. Ello afecta negativamente la estabilidad de playas [2] y poblaciones aledañas a estas costas, así como la economía de la región [3].

Ahora bien, existen técnicas no estructurales o blandas utilizadas para el manejo de la erosión costera como: regeneración de dunas, conservación y siembra de mangle y plantación o estabilización de vegetación [4]. En su implementación, se pueden utilizar especies vegetales no convencionales como Chrysobalanus Icaco, que posee potencialidades para disminuir los efectos secundarios de los fenómenos erosivos y ha sido foco de investigaciones que destacan los beneficios en la salud, industria, ambiente, entre otros [5]. Dado que la especie se desarrolla en costas del departamento y su potencial aprovechamiento económico, surgió el interés de investigar la planta para aprovechar especialmente los beneficios ecológicos (hábitat de especies migratorias, cortina rompe vientos, formación de dunas costeras, protección del suelo, mitigación de la erosión costera) y beneficios agroindustriales (pulpa, jugos de frutas, jaleas, dulces y mermeladas) [6]. Adicionalmente, la preocupación por la búsqueda de soluciones sostenibles que generen menor impacto a ecosistemas; motivó a desarrollar un proyecto en el que se utilizaran plantas nativas para afrontar problemáticas ambientales del departamento.

\section{Metodología}

\section{Área de Estudio}

La ciudad de Cartagena está localizada en el norte del departamento de Bolívar, a la orilla del Mar Caribe. En concreto, a 10을' 30" de latitud Norte y 75 32' 25" de longitud oeste respecto al Meridiano de Greenwich. Limita con el mar Caribe al Oeste y Norte, al Sur con los municipios bolivarenses de Turbaco, Turbana y Arjona, y al oriente con los de Santa Rosa de Lima, Clemencia y Santa Catalina [7]. 
Figura1. Localización de área donde se encontró la especie Chrysobalanus Icaco en el Dpto de Bolívar

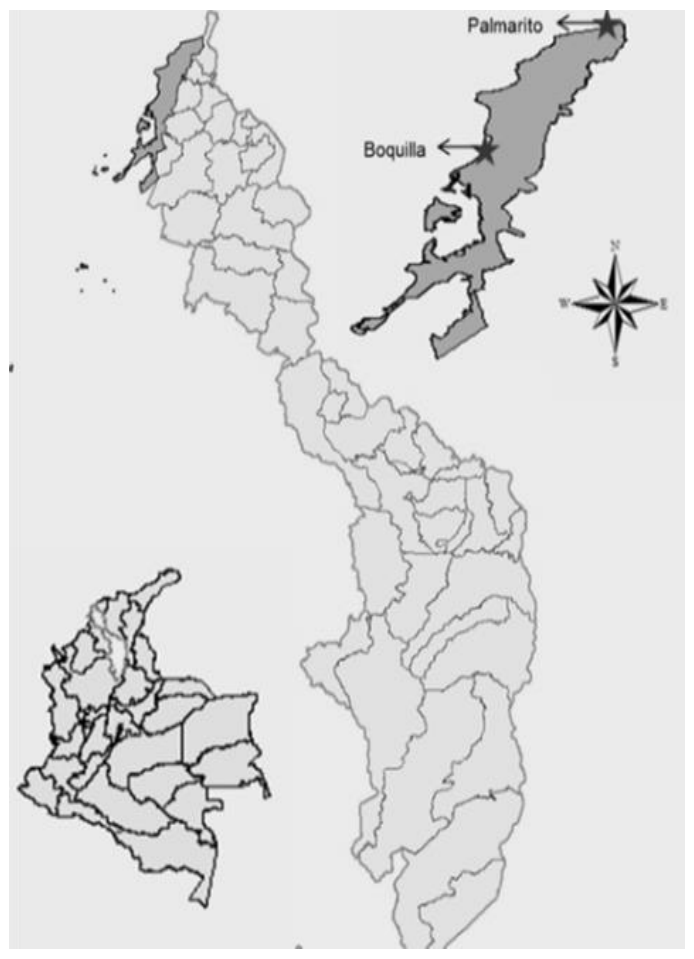

La metodología desarrollada en la investigación contempló métodos y técnicas para los periodos en los que se aplicaron, así como investigación de campo e investigación descriptiva, esta última en el proceso de caracterización cualitativa de la especie y el análisis de su desarrollo en diferentes sustratos, e investigación experimental (aplicada en ensayos de crecimiento de la especie).

La documentación, que constituyó en sí una primera fase, se realizó a partir de investigaciones de la especie Chrysobalanus Icaco, consultando estudios relacionados con información ambiental; $y$, a fin de caracterizarla cualitativamente, también se consideraron aspectos como: características generales, silvicultura, fenología, servicios ambientales ofrecidos, hábitat y vegetación asociada, entre otros. La segunda fase o de campo contempló la toma de coordenadas geográficas y parámetros ambientales específicos in situ $\left(\mathrm{pH}\right.$, temperatura del suelo $\left({ }^{\circ} \mathrm{C}\right)$, temperatura ambiente $\left({ }^{\circ} \mathrm{C}\right)$, humedad relativa, (\%)), obteniendo así las condiciones ambientales donde se desarrolla el Chrysobalanus Icaco. De hecho, la toma de muestras de suelo se dio a partir de la identificación de la especie en las zonas de la Boquilla y Palmarito. Posteriormente, fueron preservadas las muestras en cadena de custodia para ensayar en laboratorio y comparar los resultados con datos de referencia tomados en dos puntos, en el departamento de Córdoba (San Antero).

En un tercer momento, las semillas de la especie fueron recolectadas, con el fin de llevar a cabo un bioensayo que permitiera conocer el tiempo de 
Carácterización de la especie Chrysobalanus Icaco como alternativa de reforestación para mitigar procesos de erosión costera. Estudio de caso sostenibilidad ambiental de la especie en el departamento de Bolívar-Colombia

germinación y crecimiento de la planta; para ello, fue necesario sembrarlas con previos tratamientos germinativos; y luego con la adición de fertilizantes a fin de monitorear su crecimiento.

El análisis de fertilidad y nitrógeno de muestras de suelos recolectadas en Palmarito y Boquilla, mediante los métodos (NTC 5350: 2005), (NTC 5526: 2007), (NTC 5349: 2008), (Walkey \& Black), (Fosfato monobásico de calcio), $(\mathrm{KCl})$ y (VCR 004 versión 2), permitieron el estudio de micronutrientes y macronutrientes disponibles en el suelo.

Para conocer la localización de la especie, se utilizaron los Software Google Earth y Arcgis 10.3, estableciendo puntos donde se encontró Icaco en el departamento de Bolívar. Finalmente, esta información seria ordenada, clasificada y presentada en el estudio para hacer válida la interpretación de resultados.

\section{Resultados y discusión}

\section{Características generales de la especie}

El Chrysobalanus Icaco se desarrolla en forma de árbol y arbusto [8], su crecimiento se presenta en forma de arbusto entre 1-3 metros de altura y hacia terrenos estables donde las condiciones son de menor estrés hídrico. Se reproduce por semillas o estacas y posee una copa esférica o globosa. Sus hojas se presentan en forma elíptica, ovalada y circular [9], sus flores son diminutas y de color blanco o crema [10], su fruto es de tipo drupa ovalada, de color verde durante la etapa de desarrollo y en la maduración presenta colores rojos, purpura y rosado, cuyo mesocarpio es de color blanco algodón, comestible, ligeramente dulce y astringente [11]. La semilla se encuentra rodeada por un endocarpio duro, delgado, de color café claro y de forma ovalada con superficie fibrosa, de tipo dicotiledóneas [12]. En plantas provenientes de semillas, el sistema radical es fuerte y profundo, el tronco se presentan de forma variable, desde recto en algunas procedencias, hasta ligeramente torcido y muy ramificado. Sus ramas son verdes cuando jóvenes, tornándose luego rojizas hasta terminar café oscuro con lenticelas cuando adulto [13]. La corteza externa es lisa, de café oscuro a gris con gran cantidad de lenticelas [14] y, en algunos casos, ligeramente escamosa con fisuras; la corteza interna es beige, rodeada por una capa delgada rojiza semejante al del ladrillo, y la madera es marrón claro, dura y pesada. En el corregimiento de Palmarito es utilizada para combustible y construcción rústica.

\section{Aspecto silvicultural}

La dispersión de la semilla se produce cuando la diáspora es transportada por gravedad, agua, murciélagos, animales domésticos y las personas 0 , bien, al ser consumida por aves, lo cual resulta de gran importancia en la dispersión [15]. La germinación es de tipo epígea [16], en condiciones normales se inicia a los 60-90 días después de la siembra. En Veracruz se ha reportado que germinan naturalmente de julio a octubre, meses que 
coinciden con la temporada de lluvias [17]. Generalmente las semillas no requieren tratamiento pre germinativo [15]; sin embargo, se puede utilizar escarificación mecánica con elementos abrasivos como papel, lija o lima, sumergir en agua hirviendo a $100^{\circ} \mathrm{C}$ por 2-4 minutos y luego dejar reposar para la siembra, también se puede realizar una raya y las semillas se pueden remojar en agua de 12 a 24 horas antes de poner a germinar [17]. Presenta un porcentaje de germinación de $36 \%$ con semillas almacenadas durante seis meses, y $23 \%$ con semillas recién colectadas [15]. Las semillas siguen siendo viables a seis meses de almacenadas, se almacenan en bolsas de papel [15]; sin embargo, pierden su viabilidad muy rápidamente por lo que no se aconseja su almacenamiento prolongado [6]. Se colectan cuando el fruto adquiere un color amarillo-rojizo brillante [15], y la recolección se hace de forma manual en el caso de los arbustos de ramas bajas. Para el caso de los árboles en que las ramas están fuera del alcance del brazo humano, existen diversas herramientas de mango largo con las que el recolector puede alcanzar los frutos desde el suelo, utilizando una vara terminada en un gancho para desprender o arrancar los frutos. La actividad de recolección termina con el transporte de los frutos a su lugar de manejo.

\section{Recomendaciones de siembra (cultivo)}

La especie se puede reproducir por semillas, método natural de reproducción que tiene diversas ventajas; sin embargo, el tiempo para alcanzar su madurez reproductiva puede tardar más de cinco años [18], manifestando desuniformidad en el material genético, debido a la condición heterocigota y alógama de la especie [19].Un medio para disminuir dicho proceso es la propagación asexual, que consiste en el enraizamiento a través de estacas de tallo, con lo que se obtienen ciertas características como la uniformidad de las plantaciones, debido a que no hay variabilidad genética. Con esta técnica se acorta el periodo juvenil, al igual que las fases de floración y fructificación al emplear material de plantas adultas [5]. La siembra para propagación sexual se hace en camas de germinación de madera o bolsa plástica con una mezcla de tierra negra, arena de río o playa y mantillo de bosque a partes iguales o 2:1:1, cuyas bolsas deben tener el mismo número de orificios y regarse con la misma cantidad de agua [20]. Para la propagación por medio asexual, se usa el mismo sustrato con estacas particularmente apicales e intermedias de $20 \mathrm{~cm}$ de largo con hojas. En este caso, se puede dejar hasta cinco hojas en la parte alta y aplicar enraizadores como auxinas tipo AIA (Ácido indol-3-acético), AIB (Ácido indol-3-butírico). Moreno-Cassasola y Dulce Mata, en el año 2002, recomendaron realizar la propagación con sustrato arenoso en el almácigo o directamente en bolsa. El trasplante se puede hacer con hidrogel en zonas abiertas de dunas semi estabilizadas con una cubierta de pasto o herbáceas, la especie prefiere zonas muy húmedas de las dunas y llegan a tolerar la inundación [17]. Deben llevarse a cabo a una distancia que varía entre los 3-5 metros. Geilfus, Frans, 1994. A los 10 meses $0.5 \mathrm{~m}$ de altura [17]. En Venezuela se ha reportado a la planta como especie susceptible a daños o enfermedades por dípteros de la 
Carácterización de la especie Chrysobalanus Icaco como alternativa de reforestación para mitigar procesos de erosión costera. Estudio de caso sostenibilidad ambiental de la especie en el departamento de Bolívar-Colombia

familia Trypetidae: mosca de las frutas del genero Anastrepha, a daños por insecto: Ceratitis capitata Wiedemann; también es hospedadera del ácaro fitófago Oligonychus bagdasariani.

\section{Bioensayo}

Para llevar a cabo el ensayo experimental, se realizó la siembra en una caja de madera que contenía el sustrato previamente preparado. Desde el período de latencia de las semillas, y durante la germinación y crecimiento de las plántulas se realizó el riego cada tres días o cuando el sustrato estuvo seco. En la cama de germinación se colocó una muestra de 30 semillas a germinar, dividas en 3 lotes de 10 semillas, y a cada lote se aplicó un tratamiento diferente para evaluar la velocidad y el porcentaje de germinación. Una vez iniciado el proceso de germinación, se empezó a monitorear el número de hojas, la altura de la plántula y el número de plántulas. Datos reportados por autores como Moreno-Cassasola \& Dulce Mata y Geilfus han documentado un periodo de germinación en un tiempo que varía entre dos y tres meses en condiciones normales. Sin embargo, en el presente estudio, se inició el proceso de germinación en un tiempo de un mes y 18 días, diferencia que se atribuyó a los diferentes tratamientos pre germinativos que aceleraron el proceso de germinación en las semillas.

El ensayo evidenció mayor cantidad de semillas germinadas al aplicar los métodos de agua hirviendo y escarificación mecánica con lija, con un porcentaje de germinación del 80\%; mientras la muestra testigo arrojó un valor del $60 \%$. Esto indica que es recomendable aplicar alguno de los métodos anteriormente expuestos para obtener mejores porcentajes de germinación. Una vez finalizada la germinación, se agregaron dos tipos de fertilizantes orgánicos a cada lote, obteniendo los siguientes resultados: el análisis de la velocidad de crecimiento de la especie Chrysobalanus Icaco en un tiempo de un mes y dos semanas, con cada tratamiento (abonos y blanco) aplicado, alcanzó valores de crecimiento significativo, teniendo en cuenta que algunos estudios señalan que el crecimiento de la especie es lento. Por esta razón, el estudio tomó las plantas que presentaron mayores crecimientos y desarrollo.

Figura 2. Gráfico de crecimiento foliar de la especie Chrysobalanus Icaco a través del tiempo

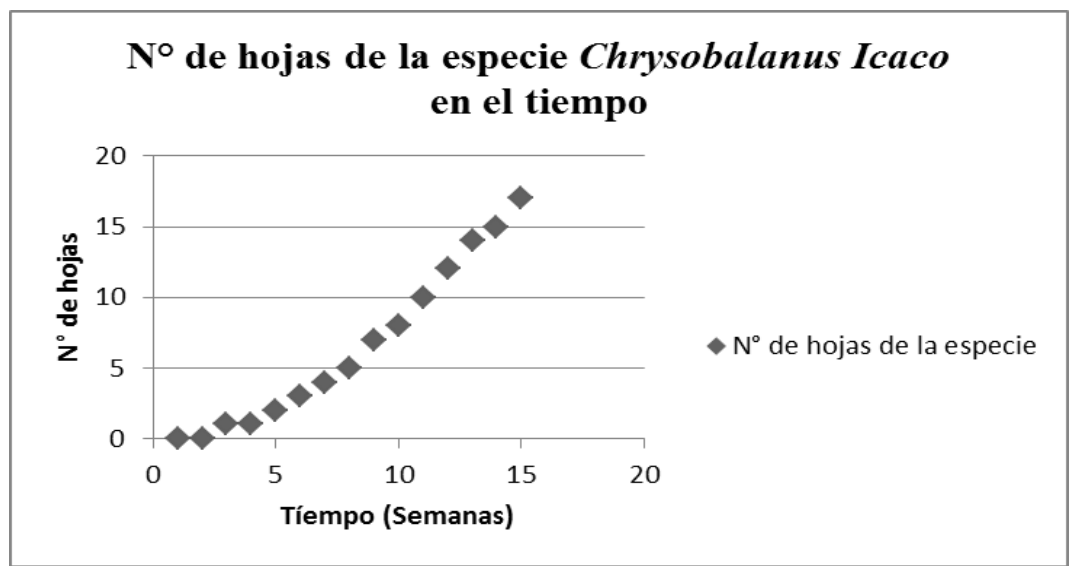


Al analizar el crecimiento foliar de la especie en condiciones normales, se evidenció un incremento, en concreto de 17 hojas para la última semana experimental. Los resultados de velocidades de crecimiento de las plantas en condiciones normales (Blanco) fueron modificándose progresivamente, a lo largo de 7 semanas consecutivas. En este tiempo, los datos de altura para la especie variaron con valores entre $0,5 \mathrm{~cm}-23,5 \mathrm{~cm}$ de altura.

Figura 3. Gráfico de velocidad de crecimiento de la especie Chrysobalanus Icaco sometida a tratamiento $\mathrm{N}^{\circ} \mathrm{A}$

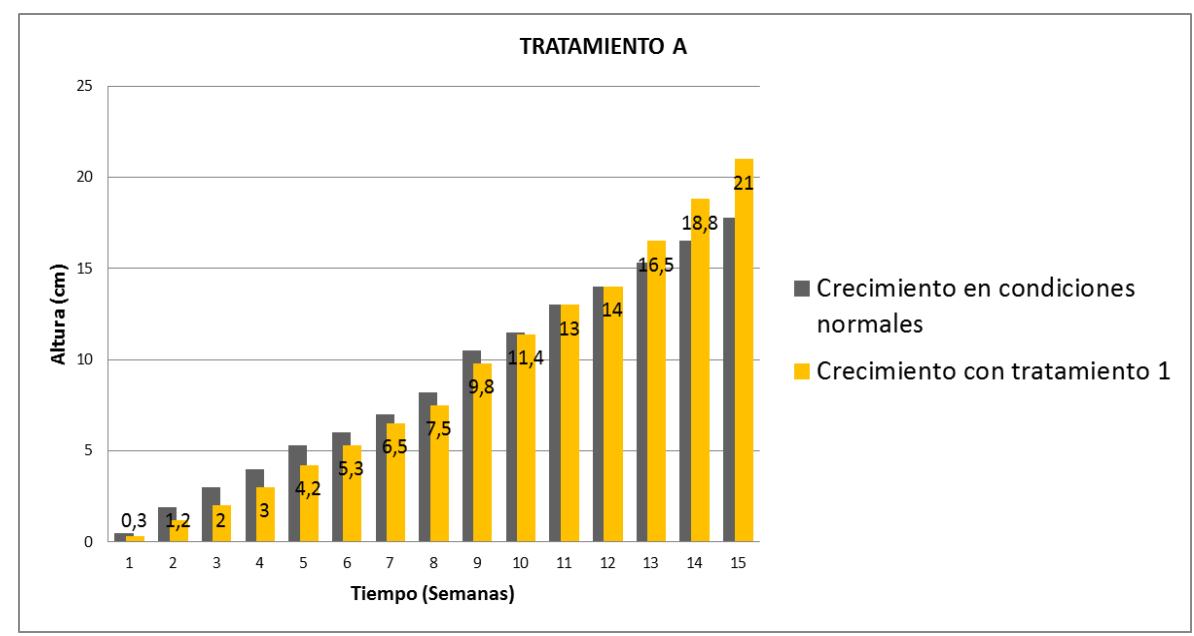

Figura 4. Gráfico de velocidad de crecimiento de la especie Chrysobalanus Icaco sometida a tratamiento $\mathrm{N}^{\circ} \mathrm{B}$

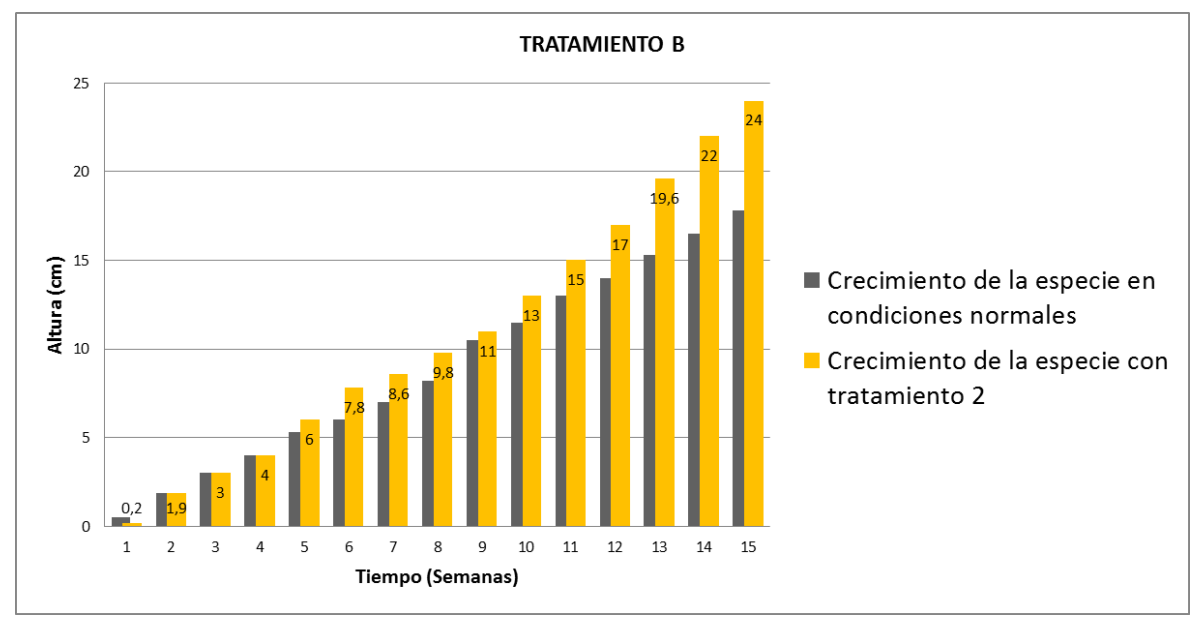

En el caso del tratamiento $A$ y $B$, las plántulas presentaron velocidades de crecimiento progresivo a lo largo de 7 semanas consecutivas. Para la semana $\mathrm{N}^{\circ}$ 9, cuando fueron aplicados los tratamientos, presentaban alturas menores a las plantas que crecían en condiciones normales, alcanzando posteriormente, valores de altura de 22 y $24 \mathrm{~cm}$, respectivamente. Las plantas a las que se les aplicaron los tratamientos A y B presentaron, entonces, niveles de crecimiento bajos, en comparación con las plantas que 
Carácterización de la especie Chrysobalanus Icaco como alternativa de reforestación para mitigar procesos de erosión costera. Estudio de caso sostenibilidad ambiental de la especie en el departamento de Bolívar-Colombia

crecieron en condiciones normales, antes de ser aplicados los tratamientos. Una vez aplicados los tratamientos, se observó, para el primer tratamiento, un incremento en las velocidades de crecimiento de las especies, alcanzando en menor tiempo la altura de las plantas ensayadas con el Blanco y, para el segundo tratamiento, sobrepasando la altura de las especies con Blanco. Resulta interesante destacar cómo las plantas que trabajaron en condiciones normales necesitaron un tiempo de 15 semanas para alcanzar una altura de $23,5 \mathrm{~cm}$, mientras que las plantas que trabajaron con tratamientos tuvieron un crecimiento más acelerado.

La aplicación del tratamiento $B$, en comparación con el tratamiento $A$, obtuvo mejores resultados en los niveles de crecimiento de esta especie, pues, permitió que la planta tuviera una altura mayor a la que crecía en condiciones ambientales normales. Por esta razón, el estudio recomienda llevar a cabo plantaciones de Chrysobalanus Icaco utilizando el tratamiento B.

\section{Fenología}

El tipo de follaje es perennifolio/caducifolio, de modo que los arboles pierden las hojas en la temporada seca y se caracterizan por tener un follaje frondoso. La floración En Tabasco-México encontrada en el calendario agronómico de floración se presenta entre Marzo y Junio [21]. En Cartagena (Colombia) para el año 2017 la época de floración de la especie se presentó entre Marzo y Mayo, correspondiendo con la época de verano. Respecto a la fructificación, en Venezuela se lleva a cabo durante los meses de Febrero a Abril, después de la época de lluvias. Allí mismo se reporta más de tres años para alcanzar producción [22], pero autores como Moreno-Cassasola \& Dulce Mata reportaron en el año 2002 que la especie alcanza madurez reproductiva a los 5 años y en Veracruz, México, el período de fructificación inicia en Agosto y termina en Enero. En Cartagena, los comerciantes reportan que el fruto se produce todo el año.

La polinización es de tipo entomofilia, llevada a cabo por insectos. En salidas de campo, se observaron abejas (Anthophila), que son atraídas por el olor de las flores al buscar alimento como polen y néctar, rico en proteínas, grasas, glúcidos y vitaminas; por otro lado, en Paraíba (Brasil) se reportó la visita de avispas de la familia Sphecidae, perteneciente al orden Hymenoptera, en flores de Icaco. 


\section{Distribución geográfica de la especie Chrysobalanus Icaco}

Figura 5. Zonas de localización de la especie Chrysoalanus Icaco en el Dpto de Bolívar

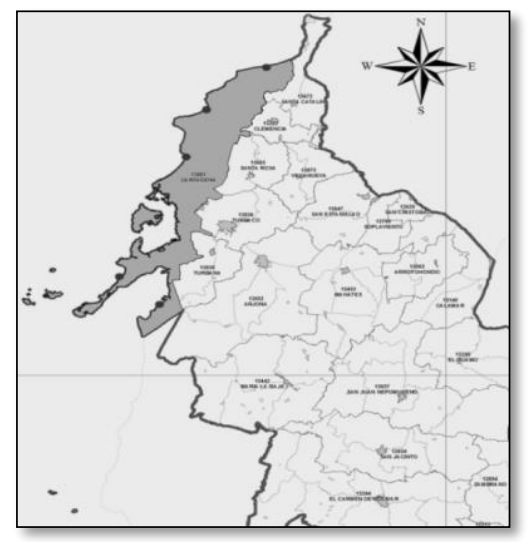

El Chrysobalanus Icaco es una especie tropical que se desarrolla y reproduce principalmente en costas. Es originaria de África y América, en África se encuentra distribuida en la parte occidental, en regiones costeras, desde Angola hasta Guinea; en el continente Americano, aparece desde Norte América, en el estado de Florida, y atraviesa las costas de Centro América [23], ubicándose en Bélice, Costa Rica, El Salvador, Guatemala, Honduras, Nicaragua, Panamá y México (Campeche, Guerrero, Oaxaca, Quintana roo, Tabasco, Tamaulipas, Veracruz). También se desarrolla en costas brasileras y de los países del norte de Sur América (Bolivia, Colombia, Ecuador, Guayana, Guayana francesa, Surinam, Venezuela). Además, se ha evidenciado la presencia del Chrysobalanus Icaco en las islas del Caribe (Archipiélago de la Bahamas, Cuba, La Española, Islas Caimán, Islas de Sotavento, Islas Vírgenes, Islas de Barlovento, Jamaica, Puerto rico, Trinidad [12]. En Colombia, la especie se encuentra distribuida en las costas de la región Caribe, región Insular, región Pacífica y en la región Andina, donde se encuentra ubicada en los bordes de ríos, hacia el territorio de bosques de galería y sabanas. Esta se ha reportado en los departamentos de Antioquia, Atlántico, Chocó, Córdoba, Cundinamarca, Huila, Magdalena, Nariño, San Andrés y Providencia, Tolima y Valle del cauca [8]. 
Carácterización de la especie Chrysobalanus Icaco como alternativa de reforestación para mitigar procesos de erosión costera. Estudio de caso sostenibilidad ambiental de la especie en el departamento de Bolívar-Colombia

En el departamento de Bolívar, la especie Chrysobalanus Icaco se encuentra, como se ha reiterado, en la Cartagena de Indias, debido a que "la mayor parte de la zona costera se encuentra en este municipio" [7], específicamente en el sector de Bocagrande y Laguito, y en los corregimientos de la Boquilla, Palmarito y Arroyo de Piedra, pertenecientes a la zona rural de la Localidad $N^{\circ} 2$ de la Virgen y Turística.

\section{Requerimientos ambientales de la especie}

\section{Clima}

Generalmente, el Chrysobalanus Icaco resiste en un clima muy cálido y seco, así como en heladas leves; también soporta inundaciones periódicas [24], pero no heladas fuertes.

La temperatura media donde generalmente se desarrolla la especie oscila entre $22-28{ }^{\circ} \mathrm{C}$ y la precipitación promedio anual es de $1000-1500$ milímetros [6]. La precipitación anual $(\mathrm{mm})$ y temperatura media $\left({ }^{\circ} \mathrm{C}\right)$ en México en las distintas regiones se da así: Noreste, 624,5/ 23,5; Centrooeste, 1296/25,2; Central-sur, 2237/27,6; Península de Yucatán-este, $1333 / 28,8$, en la región Caribe, 570/25,4, en Puerto Rico, de 500-1600/28 cálido, y en Cartagena de 1021/32,7.

La temperatura ambiente $\left({ }^{\circ} \mathrm{C}\right)$ en el corregimiento de Arroyo de Piedra durante el año 2015 fue de 36.5; en Palmarito, para el 2017 fue de 24.4; en la Boquilla para el año 2015 fue de 34.9 y para el año 2017, de 32 y en Boca Grande para el año 2017, de 25.3. La Humedad relativa en Palmarito fue de 73.5, en la Boquilla para el año 2017 fue de 64.8 y en Boca grande de 72.5. Generalmente en el departamento de Bolívar, la especie se reportó en temperaturas que oscilan entre 24,4 y $36,5^{\circ} \mathrm{C}$; mientras que la humedad relativa varía entre 64,8 y $73,5 \%$.

\section{Altitud}

Los estudios han reportado datos de altitud en diferentes regiones donde la especie se desarrolla en óptimas condiciones, así: en el estado de VeracruzMéxico, la especie se desarrolla a una altitud que varía entre los 5-150 msnm [12], y en Cartagena, Bolívar, se reporta la especie en altitudes desde los 500-2 msnm.

\section{Suelo}

La especie suele ser abundante en suelo rocoso o seco, donde generalmente otro tipo de planta está sujeta a estrés hídrico. Se desarrolla bien en suelos arenosos y salinos, bien drenados, incluso en subsuelos de drenaje pobre [6]. Usualmente, la especie también puede crecer tanto en suelos muy pobres (arena) como en suelos ricos. Crece bien en las arenas casi puras y en los sustratos rocosos a lo largo de la costa, y puede sobrevivir en los suelos calcáreos, incluyendo la piedra caliza oolítica y en los suelos secos o muy húmedos derivados de rocas ígneas, siempre que estos sitios tengan un buen drenaje. Por supuesto, crece de mejor manera en las arenas Margoza, bien 
drenadas, con valores de pH de más de 7,5 [24]. En el caso del departamento de Bolívar, la especie en desarrollo se reportó en suelos con pH básicos, con tendencias a ser alcalinos y temperatura del suelo entre 28,9 y $39.3^{\circ} \mathrm{C}$.

Tabla 1. Reporte del parámetro pH en suelos donde se desarrolla la especie Icaco en el departamento de Bolívar

\begin{tabular}{|l|l|l|l|l|l|}
\hline \multirow{2}{*}{ PARÁMETROS } & \multicolumn{4}{l}{ CORREGIMIENTOS } \\
\cline { 2 - 7 } & Arroyo de Piedra & Palmarito & \multicolumn{2}{l|}{ Boquilla } & Boca Grande \\
\cline { 2 - 7 } & 2015 & 2017 & 2015 & 2017 & 2017 \\
\hline $\mathrm{pH}$ & 10.1 & 8.6 & 7,9 & 8.1 & 7.8 \\
\hline Temperatura del suelo $\left({ }^{\circ} \mathrm{C}\right)$ & 32.5 & 28.9 & 39.3 & 36 & 33.6 \\
\hline
\end{tabular}

\section{Característica Químicas}

Figura 6. Concentraciones de pH encontrados en el suelo (dptos de Bolívar y Córdoba)

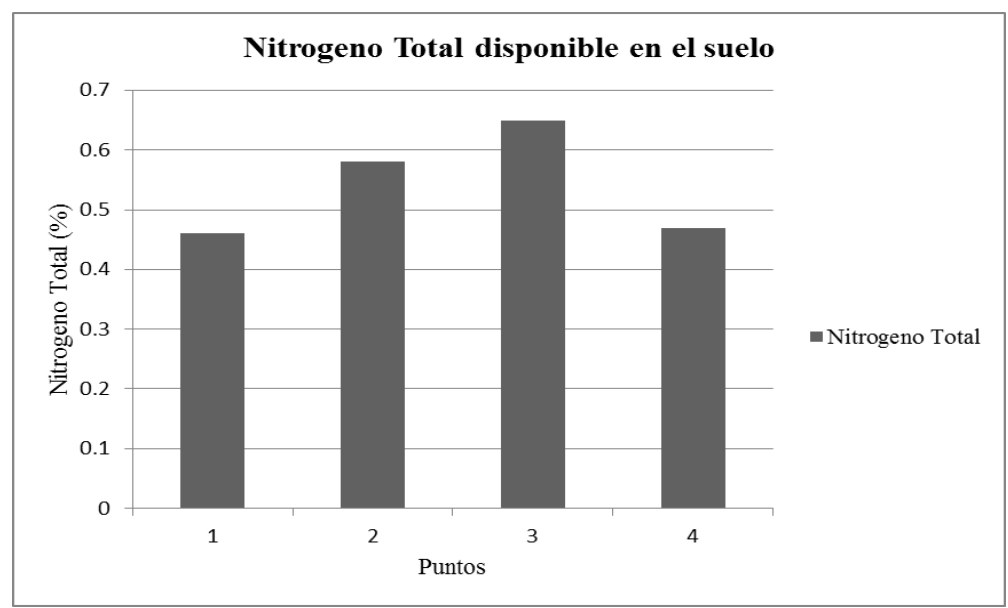

El análisis de fertilidad realizado arrojó que la especie estudiada se desarrolla en suelos muy alcalinos con $\mathrm{pH}$ entre 8.3-9.3 correspondientes a los corregimientos de la Boquilla y Palmarito, respectivamente. Comparando estos valores con datos del municipio de San Antero-Córdoba, se encontró que el $\mathrm{pH}$ en este departamento también se encuentra en un rango muy alcalino $(8.49-8,59)$ con relación al departamento de Bolívar, mostrando condiciones edafológicas semejantes en este parámetro.

Figura 7. Gráfico de concentraciones de Materia Orgánica en el suelo (dptos de Bolívar y Córdoba)

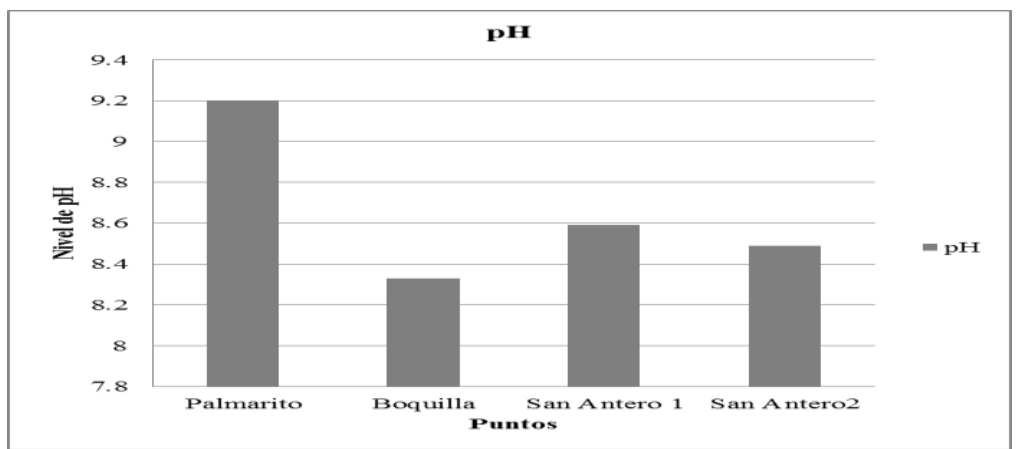


Carácterización de la especie Chrysobalanus Icaco como alternativa de reforestación para mitigar procesos de erosión costera. Estudio de caso sostenibilidad ambiental de la especie en el departamento de Bolívar-Colombia

Figura 8. Gráfico de concentraciones de Nitrógeno total en el suelo (dptos de Bolívar y Córdoba)

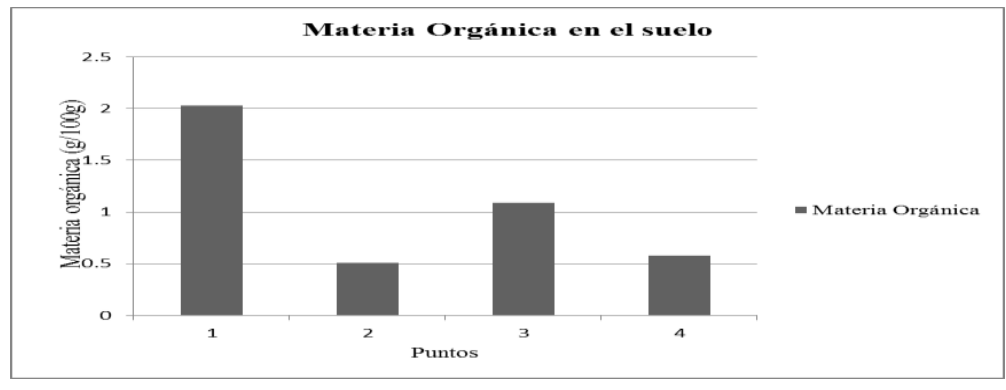

Teniendo en cuenta que el punto de estudio en Palmarito es un bosque con residuos de material vegetal, con temperatura ambiente de $24,4{ }^{\circ} \mathrm{C}$ y temperatura del suelo de $28,9^{\circ} \mathrm{C}$, se esperaría que los valores de materia orgánica sean más altos porque los procesos de degradación son lentos; sin embargo, los datos obtenidos indican que la materia orgánica en la zona es media. En climas cálidos la degradación de la materia orgánica se acelera como producto de las altas temperaturas y mucha parte de esta se volatiliza rápidamente, por lo que en las demás zonas este parámetro se presentó en niveles bajos. En este sentido, se aclara que la especie reportada se encontró en el patio de una vivienda donde recolectan los residuos vegetales del árbol.

Por su parte, el Nitrógeno total en el suelo de Palmarito (Bolívar) se encontró en un nivel alto en comparación con los demás puntos; ello se debe a que este parámetro tiene relación con la materia orgánica, cuyo contenido es de nivel medio en la zona, lo que se atribuye a la gran cantidad de material vegetal acumulado en la zona, las bajas temperaturas gracias a la altura del dosel del bosque y niveles de humedad que desaceleran el proceso de descomposición de la materia, permitiendo una mayor concentración de nitrógeno total.

Los niveles de materia orgánica encontrados en el suelo del punto de la Boquilla en el departamento de Bolívar y en San Antero (departamento de Córdoba), se encontraron en niveles bajos a altas temperaturas, por lo que se esperaría que las concentraciones de Nitrógeno también se presentaran en niveles bajos teniendo en cuenta que el clima del caribe es cálido. Sin embargo, existen controversias al respecto, pues aún no se sabe a ciencia cierta cuál o cuáles son los factores que influyen y determinan el mayor contenido de Nitrógeno y su mantenimiento en suelos salinos, razón por la cual los niveles de Nitrógeno presentes en los puntos de estudio (altos) pueden ser una respuesta al aumento de este parámetro con presencia de materia orgánica baja y temperaturas altas [25]. 
Figura 9. Gráfico de concentraciones de micronutrientes en el suelo (dptos de Bolívar y Córdoba)

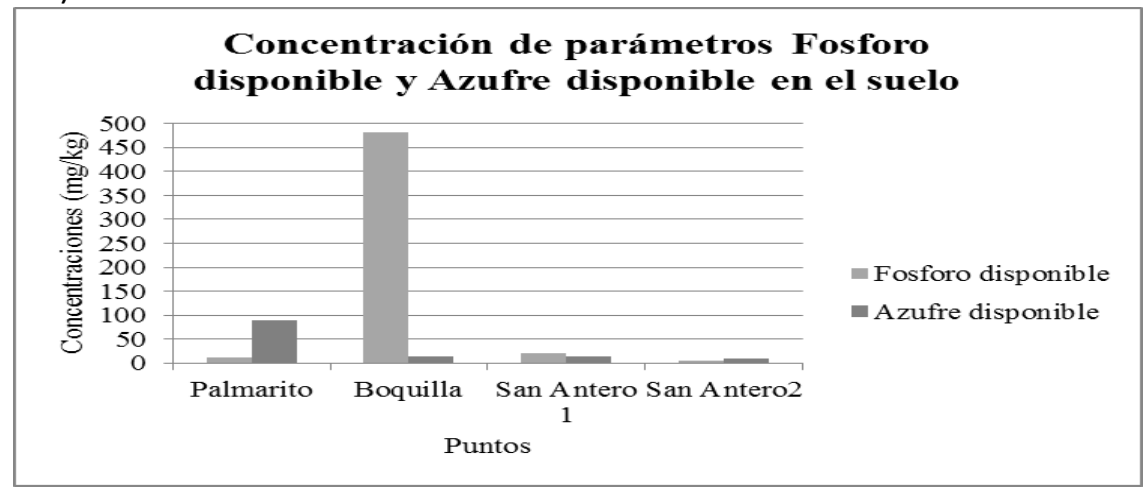

Figura 10. Gráfico de concentraciones de Fósforo y Azufre disponible en el suelo (dptos de Bolívar y Córdoba)

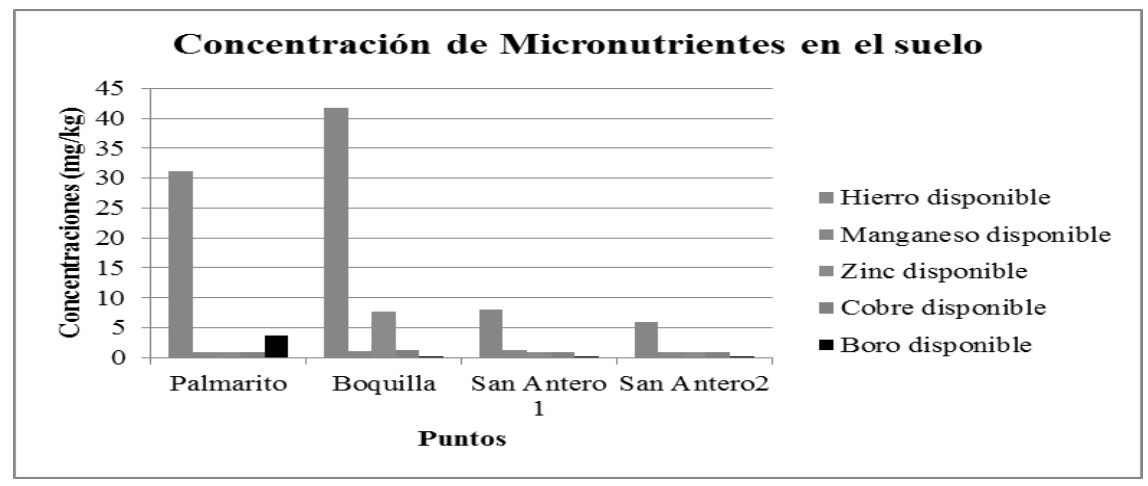

Las concentraciones de Fósforo presentes en las dos zonas estudiadas mostraron una dinámica variable del nutriente, la cual está influenciada por las propiedades del suelo, la planta y las condiciones ambientales. El nivel de Fósforo fue bajo para el Punto 1 de Bolívar (Palmarito) y para el Punto 2 de Córdoba (San Antero 2). Esto puede atribuirse a que el rango de $\mathrm{pH}$ óptimo para la disponibilidad máxima del Fósforo es de 6.0-7.0 y a que el pH de la zona es muy alcalino $(9,20)[26]$. También se debió posiblemente a las altas concentraciones de Calcio intercambiable (Ca). Por su parte, el nivel de Fósforo en los puntos 2 de Bolívar (Boquilla) y Punto 1 de Córdoba (San Antero 1) mostraron resultados en niveles alto y medio, respectivamente. Las altas concentraciones del nutriente se asociaron con fragmentos $y$ acumulación en el suelo y el mar, a través del tiempo, de restos de animales como pescado, crustáceos, entre otros [25].

Por su parte, los niveles de Azufre disponible en las zonas de estudios alcanzaron parámetros alto y medios, lo que se debió posiblemente a la interacción de restos vegetales, organismos microbianos y animales que se incorporan al suelo. Es importante destacar que en torno a las zonas de estudio se encuentran suelos con fenómenos de diapirismo, lo que también pudo influir en los niveles de este nutriente. Sin embargo, en Latinoamérica 
existe escasa información sobre la razón de presencia de Azufre en suelo, lo que se convierte en un factor limitante para el presente trabajo [25].

La concentraciones de Hierro en el suelo del departamento de Bolívar es de nivel medio con valores que oscilan entre $36,15 \mathrm{mg} / \mathrm{kg}$ (Palmarito) y 41,82 $\mathrm{mg} / \mathrm{kg}$ (Boquilla), y en el departamento de Córdoba, de un nivel bajo, presentándose valores de $8,08 \mathrm{mg} / \mathrm{kg}$ en el Punto 1 y $5,93 \mathrm{mg} / \mathrm{kg}$ en el Punto 2. Por su parte, los niveles de Manganeso en los suelos de los dos departamentos se encuentran en un nivel bajo, mostrando un comportamiento constante. De todos los puntos, la zona de Palmarito presentó un nivel muy bajo $(<1,00 \mathrm{mg} / \mathrm{kg})$ de Manganeso; en la Boquilla fue de $1,18 \mathrm{mg} / \mathrm{kg}$, y en Córdoba el Punto $1 \mathrm{de} 1,28 \mathrm{mg} / \mathrm{kg}$ y el Punto 2 de 1,01 $\mathrm{mg} / \mathrm{kg}$.

En el departamento de Bolívar, corregimiento de Palmarito, se presentaron valores de Zinc menores de $1(<1,00 \mathrm{mg} / \mathrm{kg}$ ) (rango bajo) y en la Boquilla el nivel fue alto $(7,75 \mathrm{mg} / \mathrm{kg})$, evidenciándose una variación significativa en estas zonas. Por el contrario, en San Antero los niveles presentes en las dos zonas son muy bajos $(<1,00 \mathrm{mg} / \mathrm{kg})$ mostrando un comportamiento similar. De las dos zonas muestreadas en el departamento de Bolívar solo en la Boquilla se evidenció la disponibilidad del micronutriente Cobre en un nivel medio $(1,27 \mathrm{mg} / \mathrm{kg}$ ) y en la otra zona (Palmarito), el nutriente fue bajo $(<1,00$ $\mathrm{mg} / \mathrm{kg}$ ); por otro lado, en el departamento de Córdoba la presencia del micronutriente en ambos puntos es muy bajo, presentándose valores menores que $1(<1,00 \mathrm{mg} / \mathrm{kg})$. En este sentido, el Boro está disponible en mayores cantidades. En las zonas de muestreo, se encontró en un solo punto perteneciente al corregimiento de Palmarito con un valor alto $(3,65 \mathrm{mg} / \mathrm{kg})$, los demás puntos presentaron un nivel medio del nutriente, encontrándose el mayor nivel de ellos en el primer punto del municipio de San Antero con un valor de 0,31 mg/ $\mathrm{kg}$, el segundo en la Boquilla, en Bolívar (0,23 mg/ $\mathrm{kg}$ ) y el tercero en el segundo punto en San Antero $(0,21 \mathrm{mg} / \mathrm{kg})$. Los valores anteriores mostraron que existe relativamente una disponibilidad de Boro alta, es decir, que el micronutriente es soluble en suelos muy alcalinos.

Figura 11. Gráfico de concentraciones de Macronutrientes en el suelo (dptos de Bolívar y Córdoba)

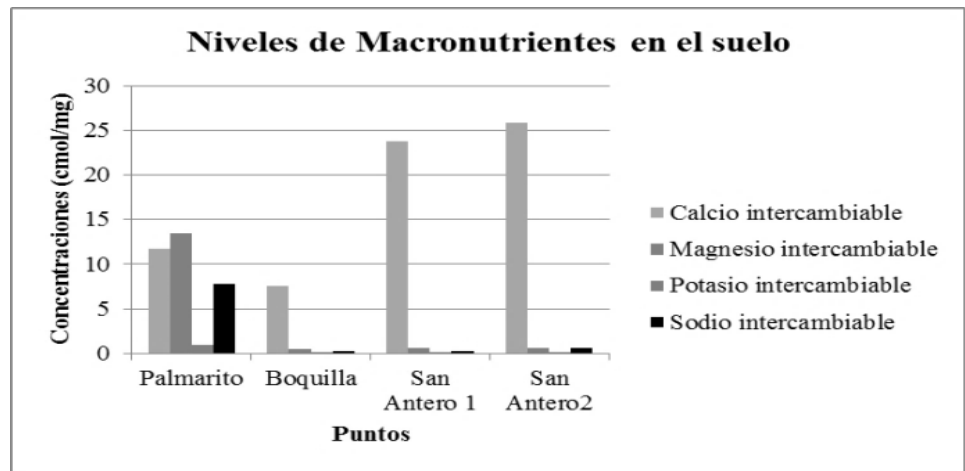


Las bajas concentraciones de Hierro disponible (Fe), Manganeso disponible $(\mathrm{Mn})$, Zinc disponible $(\mathrm{Zn})$ y Cobre disponible $(\mathrm{Cu})$ en las zonas de estudios, se atribuyen a altas concentraciones de $\mathrm{pH}$, que conforman suelos alcalinos, a la baja materia orgánica (Mo), al alto Calcio intercambiable (Ca) y a suelos livianos color blanco, que originan insolubilidad y, como consecuencia, la baja disponibilidad de nutrimentos para las plantas.

Las muestras tomadas en el departamento de Bolívar, en comparación con las de Córdoba, tienen variaciones significativas en concentraciones de Calcio, mostrando para el primer punto (Boquilla), perteneciente a Bolívar, un valor alto de $7,61 \mathrm{cmol}(+) / \mathrm{kg}$ y $11,75 \mathrm{cmol}(+) / \mathrm{kg}$ para Palmarito; de igual modo, para el departamento de Córdoba, los valores son altos, encontrándose $23,80 \mathrm{cmol}(+) / \mathrm{kg}$ en el primer punto y $25,87 \mathrm{cmol}(+) / \mathrm{kg}$ en el segundo punto.

Siguiendo con nuestra exposición, en Palmarito, en el departamento de Bolívar, el Magnesio fue alto $(13,47 \mathrm{cmol}(+) / \mathrm{kg})$ y en la Boquilla fue bajo $(0,46 \mathrm{cmol}(+) / \mathrm{kg})$. Esto muestra una diferencia significativa para el departamento de Córdoba, donde se presentó el mismo comportamiento, encontrándose un nivel de Magnesio bajo en el primer punto $(0,61$ $\mathrm{cmol}(+) / \mathrm{kg})$ y un nivel alto en el punto $2(25,85 \mathrm{cmol}(+) / \mathrm{kg})$.

El Potasio en el Punto 2 (Boquilla) presentó un valor de $0,11 \mathrm{cmol}(+) / \mathrm{kg}$ ) y en el Punto 1 (Palmarito), 0,06 $\mathrm{cmol}(+) / \mathrm{kg}$ ). En el punto $\mathrm{N}^{\circ} 1$ y 2 del departamento de Córdoba se presentaron valores menores que $1<0,06$ $\mathrm{cmol}(+) / \mathrm{kg})$, reportándose en un nivel bajo; por lo tanto, el único punto alto se presentó en el corregimiento de Palmarito en Bolívar. En el corregimiento de la Boquilla, el Sodio intercambiable presentó un nivel de $0,23 \mathrm{cmol}(+) / \mathrm{kg}$, categorizado como normal, al igual que los dos puntos en San Antero, donde se encontró en el Punto1, $0,23 \mathrm{cmol}(+) / \mathrm{kg}$ y en el Punto $2,0,62 \mathrm{cmol}(+) / \mathrm{kg}$; el nivel más alto presentado se obtuvo en Palmarito-Bolívar con 7,76 $\mathrm{cmol}(+) / \mathrm{kg}$. Por otro lado, las concentraciones de Calcio intercambiable (Ca), Potasio intercambiable (K), Magnesio intercambiable (Mg) y Sodio intercambiable $(\mathrm{Na})$ mostraron niveles de concentraciones variables para cada zona de estudio; los contenidos bajos de Magnesio y Potasio encontrados en el corregimiento de Boquilla y en San Antero se deben a las altas concentraciones de calcio.

El Magnesio intercambiable en el punto de Palmarito presentó un valor alto, lo que se debió al pH de la zona, siendo este alcalino; sin embargo, la especie se puede desarrollar en suelos donde se presenten concentraciones de macronutrientes con niveles altos, como fue el caso de Palmarito.

\section{Hábitat y vegetación asociada / zona ecológica}

El Chrysobalanus Icaco tiene una amplia plasticidad para establecerse en diversas asociaciones vegetales como selvas bajas y manglares, es común en 
áreas costeras, en dunas, playas arenosas, matorrales de playa o rastrojos [8], a'si como en selvas de galería, en bosques húmedos, muy húmedos y secos [6], en márgenes de lagos o, menos frecuentemente, tierra adentro en sabanas o vegas extensas de ríos [8]. La especie se puede encontrar en asociación con otras plantas, dependiendo de las regiones geográficas. Así, en México, en lugares donde la vegetación predominante es sabana y matorral de duna costera se le ha encontrado asociado con Nanche (Byrsonima crassifolia); cuando se encuentra en vegetación de selva baja inundable se asocia con Dalbergia glabra y Acoelorhaphe wrighti; en selva mediana subperenifolia, con la familia Palmae; en vegetación riparia y manglares, con Rhizophora mangle, Conocarpus sp, laguncularia sp; y dentro de selvas bajas caducifolias, selva mediana subcaducifolia y en el ecotono entre duna costera y selva mediana, se encuentra junto a Randia sp, Chiococca sp y Flaveria. En el bosque seco tropical se halla en la planicie del Caribe colombiano: la especie crece junto a almendro (Terminalia catappa), uvita de playa (Coccoloba uvifera), cedro (Cedrela odorata) y matarratón (Gliricidia sepium); y a nivel local, en el corregimiento de Palmarito en la ciudad de Cartagena, se encuentra asociada a uvita de playa (Coccoloba uvifera), mangle zaragoza o mangle botón (Conocarpus erectus); palma de coco (cocos nucifera). Pero principalmente, la zona ecológica donde se presenta la especie es el trópico seco y el trópico húmedo.

\section{Efecto restaurador / Servicio al ambiente}

Entre los efectos restauradores proporcionados por la especie se destaca la cobertura de hojarasca, la cual provee al suelo de abono verde, ayudando a mantener la humedad del suelo y los nutrientes. A su vez, el follaje aumenta el humus y reduce las altas temperaturas debajo del mismo. Otro efecto es la conservación del suelo y control de la erosión, la especie Chrysobalanus Icaco brinda en efecto protección al suelo, disminuyendo la erosión por el crecimiento en colonias paralelas a la costa, formando una barrera natural; estas retienen el material, creando montículos de arena que posteriormente contrarrestan el efecto nocivo del viento y de las mareas, lo cual evita las pérdidas de territorio; es decir, la especie actúa como elemento fijador de las dunas.

Figura 12. Cobertura de hojarasca en la zona de establecimiento de la especie

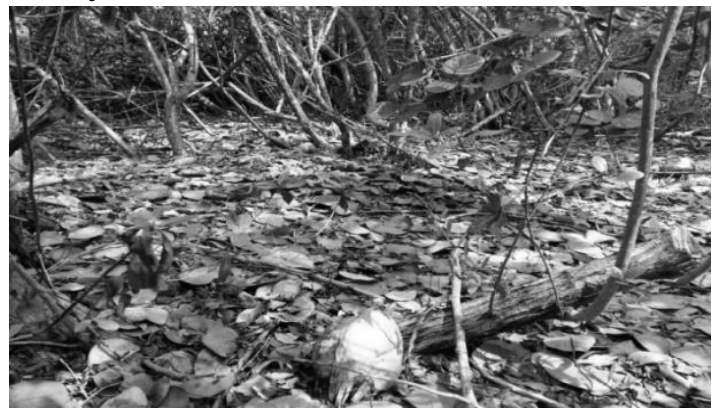




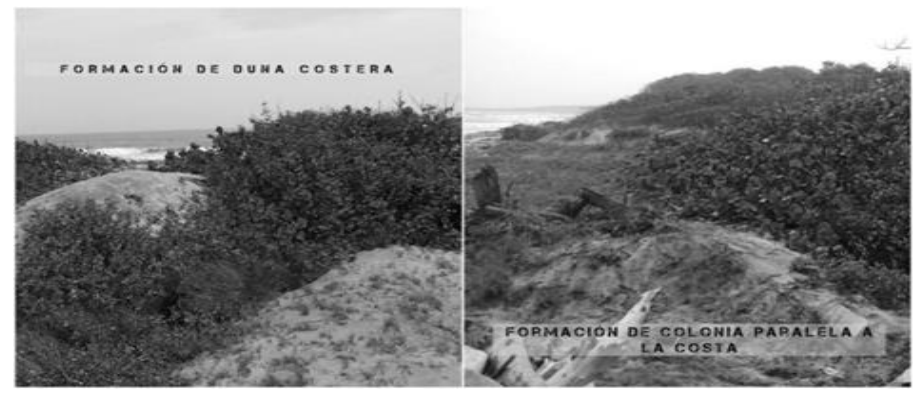

\section{Servicio}

Un aspecto relevante de este tipo de especie son los servicios ambientales que presta a la sociedad. El más importante es su función como barrera rompevientos, que evita la acción nociva del viento y las mareas, permitiendo, en cambio, que la línea de costa tenga una menor dinámica. Se ha sugerido que la conservación de esta comunidad vegetal ofrece protección a las construcciones en caso de tormentas tropicales y huracanes. De manera ornamental, como cerca viva por la belleza de sus flores de color blanco y el follaje frondoso, es usada como infraestructura de delimitación del territorio, en municipios de departamentos costeros como San Antero-Córdoba y Rincón del Mar-Sucre. También se utiliza la especie de forma ornamental como cerca viva o seto en restaurantes, cabañas y para delimitar los predios o senderos en centros recreacionales. Por último, proporciona Sombra / Refugio / alimento, la especie es ideal para sombreado permanente, debido a que tiene un amplio follaje y muchas hojas. Recupera la belleza natural del paisaje, sirve de refugio y alimento para la fauna.

\section{Usos ecológicos de la especie Chrysobalanus Icaco}

La especie Chrysobalanus Icaco es hábitat de numerosas especies de insectos, reptiles, aves, abejas y mamíferos, a los cuales brinda refugio, alimento y protección. Como arbusto aislado proporciona cobertura para que las aves aniden. Es hábitat de aves migratorias como palomas, tórtolas, loros, carpinteros, sinsontes, colibríes y otros mímidos. Sirve de alimento a la avifauna, los frutos más pequeños son consumidos por la paloma coronita (Patagioenas leucocephala) y otras palomas y tórtolas, así como por loros. Los frutos más grandes pueden ser consumidos por aves más pequeñas que los picotean e ingieren la pulpa en pedazos [26]. Por otro lado, esta especie pionera de las costas intercepta el movimiento del viento y la arena, dando inicio a la fijación de las dunas; permite así la acumulación de arena o materia orgánica y, por ende, la formación o estabilización del suelo. De esta manera se evita la erosión, principal factor que destruye las edificaciones de las costas arenosas.

\section{Experiencias con la planta}

Esta especie multipropósito puede presentar grandes expectativas de uso en sistemas agrosilvopastoriles y silvopastoriles. Se ha encontrado en huertos familiares en Palmaritico-Sucre, asociada a cultivos mixtos (plátano, 
Carácterización de la especie Chrysobalanus Icaco como alternativa de reforestación para mitigar procesos de erosión costera. Estudio de caso sostenibilidad ambiental de la especie en el departamento de Bolívar-Colombia

guayaba, yuca, mango) y, como ya se ha dicho, cumpliendo la función de setos o arbustos ornamentales, delimitando restaurantes o cabañas en San Antero-Córdoba y Rincón del mar-Sucre; debido a que, es un atractivo original.

\section{Reforestación}

La especie estudio cuenta con un potencial para reforestación productiva en zonas costeras, ya que es multifuncional e ideal para reforestar ecosistemas de playas y dunas degradadas, y para la conservación de la biodiversidad, como lo han documentado experiencias significativas con la planta que fueron determinantes para el presente estudio, en países como Puerto rico, México, Colombia y Cuba. En concreto, se han documentado estudios de bioingeniería para reforestación con Chrysobalanus Icaco, a fin de disminuir la erosión en costas, introduciendo la especie en pendientes pronunciadas, y densos matorrales de esta especie ya se han establecido en muchas cuestas empinadas de erosión [27].

Un estudio aplicado en la Costa Norte del puerto de Veracruz, utilizando métodos de restauración natural o mixta concluyó que si las dunas costeras artificiales son reforestadas con vegetación de dunas como Chrysobalanus Icaco, se acelera la estabilización sin costos de reparación, rehabilitando las dunas degradadas y recuperando los servicios del ecosistema y de protección de la costa [28]. En Veracruz, se trabajó a propósito con restauración de dunas; proponiéndose un índice de diagnóstico que permitió determinar la necesidad y las posibilidades de realizar acciones de restauración en cordones de dunas ubicados en las playas de estas costas. Se encontró que especies como Chrysobalanus Icaco producen las condiciones ambientales favorables para las especies de amplia distribución, principalmente en el centro del Golfo de México, sobre todo en las costas de Veracruz y en la zona central de la costa de Campeche.

La comisión Nacional forestal junto con la Secretaria de Medio Ambiente y Recursos Naturales de México creo un catálogo de las especies para reforestación de las dunas de la costa central de Veracruz. Entre las especies mencionadas se encuentra el Chrysobalanus icaco [18]. En Colombia, fue documentado un estudio en el marco del cual La ONG campesina y ambiental de Palomino, con el apoyo de CORPOGUAJIRA, ha sembrado alrededor de 18.000 plantas de Chrysobalanus Icaco en $22 \mathrm{~km}$ de costa, en el departamento de la Guajira, utilizándolas como protector de la rivera y obteniendo experiencias significativas [29]. En el Parque Nacional "Alejandro de Humboldt", ubicado en Cuba, se documentaron dos actividades: una de conservación y protección de cuencas hidrográficas y otra de reconstrucción de zonas costeras, utilizando la especie Chrysobalanus icaco (icaco). En este último caso, la reforestación se realizó con especies propias del lugar y de alto valor para la conservación de la biodiversidad [30]. 
Una desventaja de llevar a cabo métodos donde se utilice la especie en estudio aparece documentada en un estudio según el que el Icaco puede formar masas densas y convertirse en invasora; además, señala este estudio, que en la Polinesia francesa la especie ha sido declarada una amenaza para la biodiversidad [31]. Posiblemente, lo anterior se deba al lugar donde la especie se reforestó, toda vez que no se ha documento que el Icaco sea nativo del continente Oceánico.

\section{Conclusiones}

Con los resultados expuestos en la presente investigación, se determinó que la especie Chrysobalanus Icaco mitiga procesos erosivos en costas, pues permite la conservación y protección de suelos mediante la optimización de cobertura vegetal de la especie para reducir impactos en el paisaje.

Las condiciones ambientales y la naturaleza de suelo del departamento de Bolívar son favorables para realizar una reproducción de la especie.

En el departamento de Bolívar las características del suelo donde se desarrolla la especie son: $\mathrm{pH}$ muy alcalinos, con alto contenido de Calcio $(\mathrm{Ca})$, Sodio $(\mathrm{Na})$, Boro (B), Azufre (S) y Nitrógeno $(\mathrm{N})$ con contenidos medios en Potasio (K), Fósforo (P) y Magnesio (Mg) y deficiencias en Hierro (Fe), Manganeso (Mn), Zinc (Zn), Cobre (Cu) y pobreza en Materia Orgánica (MO) 4. Las semillas de Chrysobalanus Icaco mostraron velocidades de germinación de 1 mes y 18 días, contados desde cuando fueron aplicados los tratamientos pregerminativos, y permitiendo concluir que el tratamiento con mayores niveles favorables fue el de escarificación mecánica con lija.

El cálculo de la dosis de aplicación de fertilizantes sin una línea base es una limitante para el estudio, debido a que no existen datos científicos sobre requerimientos nutricionales y rendimientos del cultivo de Chrysobalanus Icaco.

La presencia de Chrysobalanus Icaco en el Corregimiento de Palmarito se encuentra asociada a relaciones simbióticas con plantas como Coccoloba uvifera, Ipomoea pes-caprae, Conocarpus eructus, formando un parche de bosque a lo largo de la costa, del cual son recolectados los frutos comercializados en la plaza de mercado de la ciudad

Las especies reportadas en el sector de Bocagrande y el Laguito son relictos de hacen muchos años, y actualmente son utilizadas en terrazas de viviendas de forma ornamental para embellecimiento del paisaje y sombra.

\section{Recomendaciones}

Como estos suelos poseen altas concentraciones de sales, se recomienda realizar aplicaciones de abonos orgánicos enriquecidos con oligoelementos como Zinc, Cobre, Hierro, Manganeso y macronutrientes: Potasio, Magnesio y Fósforo; además de mayores concentraciones de Materia Orgánica que eleven las condiciones de los nutrientes que se encuentren en niveles bajos 
en el suelo, para contribuir al desarrollo de la planta y mejorar la cosecha. 2. Llevar a cabo un estudio de caracterización física del suelo que permita establecer su textura, estructura, porosidad y permeabilidad para cultivos agrícolas. 3. Se recomienda llevar a cabo ensayos experimentales con la especie, realizando plantaciones en ecosistemas degradados para evaluar los efectos restauradores. 4. Hacer un estudio de la relación duna-planta en Palmarito-Bolívar, donde se ha encontrado este ecosistema (sistema dunar) en buen estado de conservación; por tanto, sería de gran relevancia estudiar uno de los pocos sistemas biológicos en su especie con que cuenta este departamento, para su preservación y conservación.

\section{Referencias bibliográficas}

1. J. R Tayupanta, La erosión. En La erosión hídrica: procesos, factores y forma. Quito, Ecuador: Sección del Comunicación del INIAP, 1993, pp. 1-17.

2. Dirección General del Observatorio Ambiental. Erosión Costera [Internet], San salvador, Salvador, Min. De Medio Ambiente y Recursos Naturales., $2014 . \quad$ Disponible: < http://www.snet.gob.sv/ver/oceanografia/seccion+educativa/erosi on+costera/>

3. S. Marcomini, \& R. López. "Erosión y Manejo Costero de Villa Gesell [Internet], Buenos aires, Argentina", Unión por Gesell Gesell., 2007. Disponible: https://digital.bl.fcen.uba.ar/gsdl282/Libro_0002_Marcomini/6_manejo_costero/index_Gesell.htm.

4. INVEMAR, Informe del estado de los ambientes y recursos marinos y costeros de Colombia[Internet], Santa Marta, Magdalena, Serie de Publicaciones Periodicas., $2017 . \quad$ Disponible: < http://www.invemar.org.co/documents/10182/14479/IER_2016_b aja.pdf/4648b25a-fb94-4294-9ecb-a027abb3a211>

5. S. Vargas, G. Arellano, \& R. Soto (1999). Enraizamiento de estacas de Icaco (Chrysobalanus Icaco L.) sometidas a aplicaciones de auxinas [Internet]. Paraíso, Tabasco,. México: Bioagro. Disponible: < http://www.ucla.edu.ve/bioagro/Rev11(3)/4.\%20Enraizamiento\%2 0de\%20estacas\%20de\%20icaco.pdf

6. A. Martinez, Y. Teran, H. Barazarte, D. Jimenez, \& R. D' Aubeterre. Propiedades fisico quimicas de la pulpa y semilla de Icaco (Chrysobalanus Icaco) para su aprovechamiento agroindustrial. Venezuela: Editorial Aportes de saberes., 2016.

7. PNUD, Plan Distrital de Gestión del Riesgo Cartagena de Indias [Internet], Carrtagena, Bolívar, Alcaldía Mayor de Cartagena de Indias., 2013. Disponible: 
http://www.dadiscartagena.gov.co/images/docs/crue/n2015/plan_ distrital_gestion_riesgo_v2_2003.pdf >

8. G. Prance, Flora de Colombia [Internet], Bogotá, D.C, Editores E.Forero, J. Betancur, G. Galeano, \& J. Aguirre., 2016. Disponible: < http://ciencias.bogota.unal.edu.co/fileadmin/content/icn/publicaci ones/floradecolombia/fdc025.pdf.pdf>

9. G. Espinoza, S. Vargas, \& M. Engleman, "Contribución al Estudio de la Anatomía Foliar del Icaco (Chrysobalanus Icaco L)", Bioagro, vol. 14, no. 1, pp. 29 - 36., 2002. Disponible: < http://www.redalyc.org/articulo.oa?id=85714105>

10. M. Lascurai, S. Avedaño, S. Del amo, \& A. Niembro, Guía de frutos silvestres comestibles en Veracruz: Chrysobalanus Icaco. Veracruz, México: A. pozo Editores., 2010.

11. J. León, Botánica de los Cultivos Tropicales Icaco. San José, Costa Rica, Editorial IICA, 1987, pp. $1-445$.

12. C. Durán-Espinoza, \& F. Lorea-Hernández, Flora de Veracruz, Xalapa, Flora de Veracruz, México D.F: Cromocolor ed., pp. 1-38.

13. A. Orellana, Catálogo Frutales Nativos de Guatemala, [Internet], 1 Edición, Ciudad de Guatemala, ICTA., 2014. Disponible: < http://www.icta.gob.gt/publicaciones/Frutales\%20nativos/Catalogo \%20de\%20frutales\%20nativos\%20de\%20Guatemala,\%202014.pdf>

14. F. Maldonado, G. Vargas,R. Molina, \& A, Sánchez. Frutales Tropicales de Tabasco [Internet], Versión 3, Tabasco; México: D.R Universidad de Juárez Autónoma de Tabasco., 2004. Disponible: < https://es.scribd.com/doc/109024901/Frutales-Tropicales-deTabasco>

15. J. Francis, "Wildland Shrubs of the United States and Its Territories: Thamnic Descriptions. [Scientific magazine] General Technical Report Station (GTR)", International Institute of Tropical Forestry, 1 (1), pp. 99-200, 2004.

16. L. Rosabal, L. Mártinez, Y. Reyes, C. Dell'Amico, \& M. Núñez, "Aspectos Fisiologicos, Bioquímicos y Expresión de Genes en Condiciones de Defícit Hídrico. Influencia en el Proceso de Germinación", Cultivos Tropicales Scielo, vol. 35, no. 3, pp. 1-5., 2014.

17. P. Moreno-Cassasola, \& I. Dulce Mata. Especies para reforestación de la dunas de la costa central de Veracruz. Zapopan, Jalisco, México, 2002. Pp. 1 -14. 
18. M. Jimenez, E. Mendez, J. Lesher, R. Molina, \& R. Hernández, "Una ventana al Estudio del Genóma del Chrysobalanus Icaco L", Tierra viva, Naturaleza, vol. 17, no. 33, p. 61-65., 2011.

19. D. kester, \& H. Hartman. Propagación de Plantas. Argentina: Editorial CECSA, 1980, pp. $25-382$.

20. R. López, C. Sarmiento, L. Espitia, A. Barrero, C. Consuegra, \& B. Gallego, Cien Plantas del Caribe Colombiano. Usar para conservar: Aprendiendo de los Habitantes del Bosque seco. Bogotá D. C, Colombia, Fondo patrimonio natural, 2016, pp. 1-24.

21. L. López-Cruz, El caco (Chrysobalanus Icaco) como cultivo potencial en zonas de trópico seco, Venezuela, 2015. Disponible: < https://www.intagri.com/articulos/frutales/el-caco-como-cultivopotencial>

22. F. Leal, \& J. Navas, Cultivos Multiestrata:un módelo de desarrollo agrícola para el área de Barlovento. Venezuela: Mracay, 2000.

23. Derechos Reservados, Recursos Genéticos de las Plantas Cultivadas de America Central. Turrialba, Costa Rica, O. IICA/CATIE Ed, 1979, pp. $1-32$.

24. F. Geilfus, El Árbol al Servicio del Agricultor. Turrialba, Costarica: O. IICA/CATIE Ed, 1994, pp. 1-656.

25. J. Francis, \& C. Lowe. (2000, Junio) “Biotecnología de Árboles Nativos y Exóticos de Puerto Rico y las Indias Occidentales Coccoloba Uvifera", USDA, vol. 1, no. 1, pp. 148-151., 2000.

26. H. Fassbender, \& F. Bomemiza, Química de Suelos con Énfasis en Suelos de América Latina. San José, Costa Rica: ICA, 1987, pp. 45-198.

27. Smart-Fertilizer Management. (----) El Fósforo en el Suelo y las Plantas , [Internet], Smart-Fertilizer Management. Disponible desde: < https://www.smart-fertilizer.com/es/articles/phosphorus >

28. BirdsCaribbean. Patrimonio Vegetal: Plantas y arboles nativos para las aves y personas del caribe, [Internet], Caribe, BirdsCaribbean., 2014. Disponible desde: < http://www.birdscaribbean.org/wpcontent/uploads/2014/06/Patrimonio-Vegetal-BirdsCaribbean.pdf >

29. F. Franco-Ospina, Etnobotánica de la Cuenca Baja del Rio Palomino, [Diapositivas]., 2010.2 Disponible: http://www.udla.edu.co/documentos/docs/Programas\%20Academi cos/Ingenieria\%20Agroecologica/Memorias/I\%20Simposio\%20Inter nacional\%20de\%20Agroecologia/Palomino\%20\%20A-Z.pdf.

30. N. Sánchez-Abad, J. Turro-Columbié, \& G. Rodríguez-Coba, Manejo de la flora en el sector Baracoa del Parque Nacional Alejandro 
Humboldt, [Internet], 2012. Cuba. Disponible desde:< http://www.radiobaracoa.icrt.cu/es/especiales/ecovida/item/4754palpan-logros-en-sector-baracoa-del-parque-humboldt.html>

31. United Nations Environment Programe. Africa Environment Outlook 2: Our Environment, Our Wealth, UNEP/Earthprint Ed., 2006. 